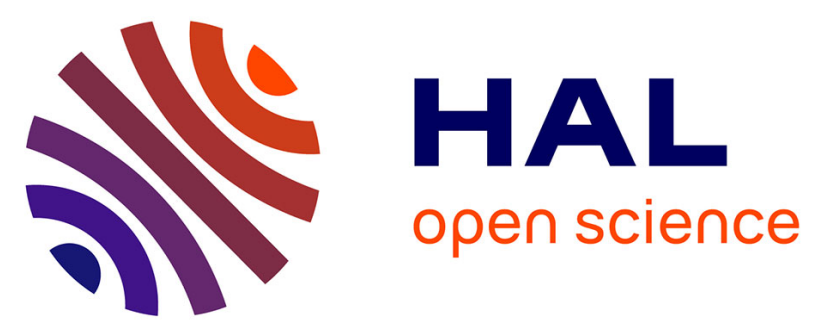

\title{
Contribution du test d'expansion d'anneaux à l'étude de différentes lois de comportement élastoplastique dans le domaine des grandes vitesses de déformation. Application au cuivre et à l'acier Mars 190
}

\author{
A. Juanicotena, F. Llorca
}

\section{To cite this version:}

A. Juanicotena, F. Llorca. Contribution du test d'expansion d'anneaux à l'étude de différentes lois de comportement élastoplastique dans le domaine des grandes vitesses de déformation. Application au cuivre et à l'acier Mars 190. Journal de Physique IV Proceedings, 1997, 07 (C3), pp.C3-541-C3-546. 10.1051/jp4:1997393 . jpa-00255550

\author{
HAL Id: jpa-00255550 \\ https://hal.science/jpa-00255550
}

Submitted on 1 Jan 1997

HAL is a multi-disciplinary open access archive for the deposit and dissemination of scientific research documents, whether they are published or not. The documents may come from teaching and research institutions in France or abroad, or from public or private research centers.
L'archive ouverte pluridisciplinaire HAL, est destinée au dépôt et à la diffusion de documents scientifiques de niveau recherche, publiés ou non, émanant des établissements d'enseignement et de recherche français ou étrangers, des laboratoires publics ou privés. 


\title{
Contribution du test d'expansion d'anneaux à l'étude de différentes lois de comportement élastoplastique dans le domaine des grandes vitesses de déformation. Application au cuivre et à l'acier Mars 190
}

\author{
A. Juanicotena et F. Llorca
}

Commisssariat à l'Énergie Atomique, Centre de Valduc, 21120 Is-sur-Tille, France

\begin{abstract}
The expanding ring test represents a new experimental technique allowing the study of high strain rate material behavior. This test allows to validate various constitutive relations for strain rate up to $10^{4} \mathrm{~s}^{-1}$, or , in addition of dynamic test like Split Hopkinson Pressure Bars bars, to calculate associated coefficients to these models for these strain rates levels. In this work, we evaluate three elastoplastic models on two materials : copper and armor steel.

Résumé. Le test d'expansion d'anneaux représente une nouvelle technique expérimentale permettant l'étude du comportement mécanique des matériaux aux grandes vitesses de déformation. Ce test permet de valider différentes lois de comportement pour des vitesses de déformation de l'ordre de $10^{4} \mathrm{~s}^{-1}$, ou alors, en complément d'essais dynamiques plus classiques par barres d'Hopkinson notamment, de déterminer les coefficients associés à ces modèles pour ces niveaux de vitesses. Dans ce travail, nous évaluons trois lois de comportement sur deux matériaux d'étude : le cuivre CuC2 et l'acier Mars 190.
\end{abstract}

\section{INTRODUCTION}

La détermination expérimentale des coefficients associés aux différents modèles de comportement dynamique des matériaux est basé sur l'interprétation d'expériences simples. Dans la plupart des cas, ces données expérimentales sont déduites d'essais de traction et de compression simple quasi-statique, et d'essais dynamiques en compression sur barres d'Hopkinson. Ce type d'essais est cependant limité aux vitesses de déformation moyennes $\left(\approx 10^{3} \mathrm{~s}^{-1}\right)$. Afin d'étendre la gamme de validité des lois de comportement, il s'avère nécessaire de développer des expériences complémentaires permettant d'atteindre des niveaux de vitesses de déformation plus élevés. Nous pourrons ainsi valider ces lois de comportement dans un domaine plus étendu, ou bien recalculer leurs coefficients dans cette nouvelle gamme de vitesse de déformation. Le test d'expansion d'anneaux fait partie de ces techniques complémentaires, avec des vitesses de déformation variant de $5.10^{3} \mathrm{~s}^{-1}$ à $10^{4} \mathrm{~s}^{-1}$, et des déformations pouvant atteindre 50\% suivant les matériaux. Dans ce travail, nous utilisons le test d'expansion d'anneaux comme moyen d'étude et de comparaisons de différentes lois de comportement élastoplastiques : Johnson-Cook, Zerilli-Armstrong et Preston-Tonks-Wallace. Ces modèles prennent en compte les effets de la déformation, de la vitesse de déformation et de l'adoucissement thermique sur la contrainte d'écoulement du matériau. Nous présentons des résultats d'essais d'expansion d'anneaux sur cuivre et acier Mars 190. Le traitement numérique de ces données (voir Partie I) permet de décrire l'évolution temporelle de l'état de chargement $(\sigma, \varepsilon, \dot{\varepsilon})$ des deux matériaux testés. Il est alors possible d'évaluer les trois modèles considérés, par comparaison des résultats théoriques et expérimentaux.

\section{LOIS DE COMPORTEMENT}

\subsection{Modèle de Johnson-Cook}

Johnson et Cook proposent une loi empirique [1]-[2], conçue à partir de résultats expérimentaux, et destinée à l'implantation dans les codes de calcul. La contrainte d'écoulement au sens de Von Misès $(\sigma)$ s'écrit :

$$
\sigma=\left[A+B \varepsilon^{n}\right]\left[1+C \ln \ddot{\varepsilon}^{\bullet}\right]\left[1-T^{* m}\right]
$$

où $\varepsilon$ est la déformation plastique équivalente, $\ddot{\varepsilon}=\dot{\varepsilon} / \dot{\varepsilon}_{0}$ le terme adimensionnel relatif à la vitesse de déformation plastique (avec $\dot{\varepsilon}_{0}=1 \mathrm{~s}^{-1}$ ), et $T^{*}$ la température "homologue" : $T^{*}=\left(T-T_{\text {ma }}\right) /\left(T-T_{\text {fac }}\right)$. 


\subsection{Modèle de Zerilli-Armstrong}

Il s'agit d'un modèle semi-empirique [3]-[4], dont les relations constitutives sont basées sur la mécanique des dislocations. L'analyse de l'activation thermique permet de prendre en compte les effets conjugués de l'écrouissage, de la vitesse de déformation, et de la température. De plus, ce modèle tient compte de la taille des grains ainsi que de la structure cristallographique, sachant que ces deux paramètres influent sur la résistance et la ductilité du matériau. La contrainte d'écoulement au sens de Von Misès s'écrit :

$$
\begin{aligned}
& \text { CFC: } \sigma=\Delta \sigma_{G}^{\prime}+C_{2} \varepsilon^{1 / 2} \exp \left(-C_{3} T+C_{4} T \ln \dot{\varepsilon}\right)+k l^{-1 / 2} \\
& \text { CC : } \sigma=\Delta \sigma_{G}^{\prime}+C_{1} \exp \left(-C_{3} T+C_{4} T \ln \dot{\varepsilon}\right)+C_{5} \varepsilon^{n}+k l^{-1 / 2}
\end{aligned}
$$

où $k$ est le facteur microstructural d'intensité de contrainte, et $\mathrm{I}$ le diamètre moyen des grains. Dans cette étude, la contribution de l'influence de la taille de grains et de la densité de dislocations sur la contrainte d'écoulement sera prise comme étant constante, et telle que : $C_{0}=\Delta \sigma_{G}^{\prime}+k l^{-1 / 2}$.

Les coefficients du modèle pour les matériaux $\mathrm{CC}$ sont $C_{0}, C_{l}, C_{3}, C_{4}, C_{5}$ et $n$; et pour les matériaux $\mathrm{CFC}, C_{0}, C_{2}, C_{3}$ et $C_{4}$.

\subsection{Modèle de Preston-Tonks-Wallace}

Preston, Tonks et Wallace ont développé au début des années 90 [5], un nouveau modèle rhéologique semi-empirique sensé être applicable à toutes températures, pressions, déformations et vitesses de déformation. Ce modèle utilise les trois variables adimensionnelles suivantes : $\hat{\tau}=\tau / G$ où $\tau$ est la contrainte d'écoulement en cisaillement pur et $G$ le module de cisaillement, $\hat{T}=T / T_{\text {fus }}$ oủ $T$ est la température et $T_{f u s}$ la température de fusion, $\hat{\dot{\psi}}=\dot{\psi} / \dot{\xi}$ où $\dot{\psi}$ représente la vitesse de déformation équivalente et $\xi=(l / 6)(4 \pi)^{1.3} \omega_{D}$ est l'inverse du temps nécessaire à une onde de cisaillement pour traverser une cellule unité du matériau ( $\omega_{D}$ : fréquence de Debye).

Dans sa formulation, le modèle distingue très clairement les vitesses de déformation moyennes $\left(\dot{\psi} \leq 10^{5-6} \mathrm{~s}^{-1}\right.$ ) pour lesquelles l'analyse de l'activation thermique est prise en compte, des grandes vitesses de déformation $\left(10^{5-6} s^{-1} \leq \dot{\psi} \leq 10^{4-12} s^{-1}\right)$ dont la contribution est évaluée de façon plus empirique et où la contrainte d'écoulement et la vitesse de déformation obéissent approximativement à une loi puissance. Le comportement élastoplastique du matériau est encadré par la limite d'élasticité $\tau_{\text {s }}$ $(\psi \rightarrow 0)$ et par la contrainte de saturation $\tau_{s}(\psi \rightarrow \infty)$ qui s'écrivent - sous forme adimensionnalisées de la façon suivante :

$$
\begin{aligned}
& \hat{\tau}_{y}=\max \left\{Y_{0}-\left(Y_{0}-Y_{\infty}\right) \operatorname{erf}[k \hat{T} \ln (\gamma / \hat{\dot{\psi}})], \min \left\{Y_{l}(\hat{\dot{\psi}} / \gamma)^{\gamma_{2}}, S_{0}(\hat{\dot{\psi}} / \gamma)^{\beta}\right\}\right\} \\
& \hat{\tau}_{s}=\max \left\{S_{0}-\left(S_{0}-S_{\infty}\right) \operatorname{erf}[k \hat{T} \ln (\gamma / \hat{\dot{\psi}})], S_{0}(\hat{\dot{\psi}} / \gamma)^{\beta}\right\}
\end{aligned}
$$

où $Y_{0}, Y_{\infty}, S_{0}, S_{\infty}, k$ et $\gamma$ sont des constantes du modèle.

La loi d'écrouissage du modèle PTW s'inspire de la loi de comportement de Voce [6] :

$$
d \hat{\tau} / d \psi=\theta_{0}\left(\tau_{s}-\tau\right) /\left(S_{0}-\tau_{y}\right)
$$

où $\theta_{0}$ est une constante du modèle et représente le taux d'écrouissage initial.

Cependant, des observations complémentaires ont montré que pour les métaux à structure cristallographique cubique faces centrées, ce taux d'écrouissage décroît moins rapidement vers zéro lorsque la contrainte de saturation augmente. Ce comportement incite PTW à modifier la loi de Voce, qui

apparaît alors sous la forme : $\quad \frac{d \hat{\tau}}{d \psi}=\theta_{0} \frac{\exp \left\{p\left(\tau_{s}-\tau\right) /\left(S_{0}-\tau_{y}\right)\right\}-I}{\exp \left\{p\left(\tau_{s}-\tau_{y}\right) /\left(S_{0}-\tau_{y}\right)\right\}-I}$

$S_{0}$ est la contrainte de saturation à $T=0$ et $p$ est une constante adimensionnelle. La contrainte d'écoulement s'obtient par intégration de (2.6) et (2.7) à température et à vitesse de déformation constante. 
Le modèle comporte donc onze coefficients adimensionnels. Dans cette étude, seule la partie moyennement dynamique sera abordé.

\section{TEST D'EXPANSION D'ANNEAUX}

\subsection{Principes et diagnostics}

Le test d'expansion d'anneau représente un moyen simple et efficace de réaliser un essai dynamique de traction uniaxiale. Le principe consiste à imposer une expansion radiale à un anneau mince, au moyen d'une onde de choc générée par explosif et transmise par l'intermédiaire d'un cylindre en acier (fig. 1). Deux anneaux de garde placés de part et d'autre de l'anneau central permettent de limiter les effets de bords dus à l'arrivée de l'onde de choc sur l'anneau et ainsi de préserver l'homogénéité des déformations au

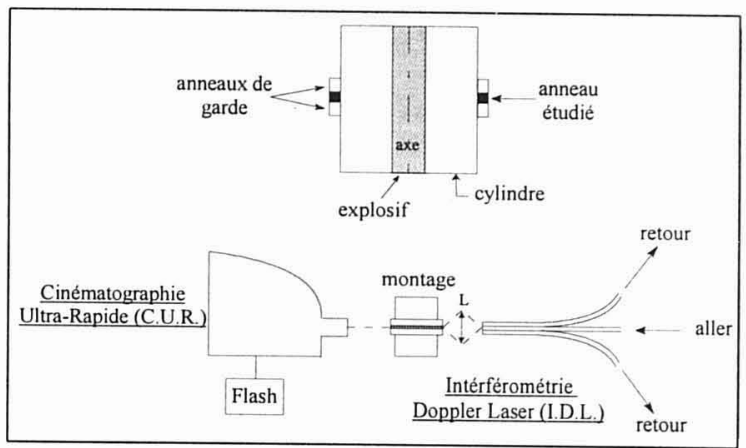

Figure 1 - Schéma de principe du test d'expansion d'anneau sein de l'anneau.

L'anneau en expansion n'est alors soumis à aucune force extérieure, et sa vitesse décroît au cours du temps sous l'action de la contrainte d'écoulement du matériau étudié. Une mesure directe de la vitesse d'expansion radiale au cours du temps est obtenue par Interférométrie Doppler Laser. En complément, une caméra mécano-optique Barr\&Stroud permet de suivre l'évolution temporelle du processus d'expansion (fig. 1). Nous pouvons faire varier la vitesse de lancement de l'anneau et donc la vitesse de déformation en jouant sur l'épaisseur de l'anneau et le diamètre de l'explosif.

\subsection{Analyse}

Les hypothèses d'uniformité des déformations au sein de l'anneau et d'uniaxialité des contraintes (contraintes tangentielles) nous permettent, grâce à l'application de la relation fondamentale de la dynamique, de déterminer les paramètres rhéologiques du matériau. Nous obtenons les relations suivantes : $\sigma_{\theta}=-\rho r \ddot{r}$ (contrainte tangentielle), $\varepsilon_{\theta}=\ln \left(r / r_{0}\right)$ (déformation vraie), $\dot{\varepsilon}=\dot{r} / r_{0}$ (vitesse de déformation), où $\rho$ est la masse volumique du matériau considéré, $r$ le rayon de l'anneau, $\dot{r}$ et $\ddot{r}$ la vitesse et l'accélération radiale, et $r_{0}$ le rayon initial. La dérivation et l'intégration du signal de vitesse obtenu par I.D.L. nous donne alors accès aux paramètres mécaniques et donc au comportement global du matériau.

Des simulations numériques du test (voir partie I) nous ont permis de vérifier les hypothèses faites quant au rôle joué par les anneaux de garde, l'uniformité des déformations et l'uniaxialité des contraintes. La fig. 2 montre un exemple d'interférogrammes obtenu sur acier Mars 190 à l'aide de caméras électroniques à balayage variable. La courbe de vitesse correspondante est présentée sur la fig. 3. Nous pouvons distinguer ici trois zones bien distinctes. Une première zone (oscillations) qui correspond aux allers retours de l'onde de choc à travers l'anneau (voir partie I), une deuxième zone où la vitesse décroît linéairement jusqu'à atteindre une valeur nulle et qui correspond à l'écoulement plastique du matériau, et enfin une troisième zone où l'anneau oscille autour d'une position d'équilibre et qui est due à la relaxation de l'énergie de déformation élastique emmagasinée par l'anneau au cours de l'essai [7].

\section{RESULTATS SUR CUIVRE ET ACIER MARS 190}

Nous proposons ici des résultats expérimentaux sur un cuivre pur, de nuance $\mathrm{CuC} 2$ et de structure cubique faces centrées, et sur un acier à blindage, l'acier Mars190, de structure cristallographique quadratique centré. Des essais quasi-statiques et dynamiques en température ont été réalisés en complément des tests 
d'expansion d'anneaux afin de déterminer les coefficients associés aux trois lois de comportement précédemment citées.

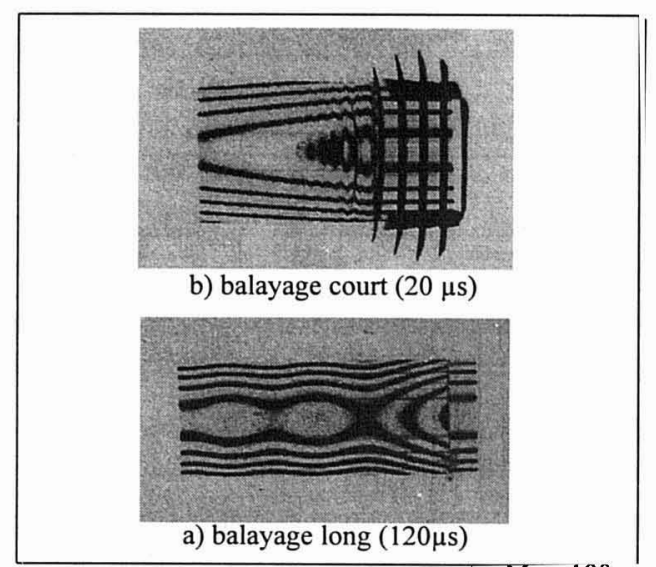

Figure 2 - Expansion a'anneaux sur acier Mars 190 épaisseur d'anneau : $3 \mathrm{~mm}$; diamètre explosif : $6 \mathrm{~mm}$ $(3 \mathrm{~mm} / \phi 6 \mathrm{~mm})$

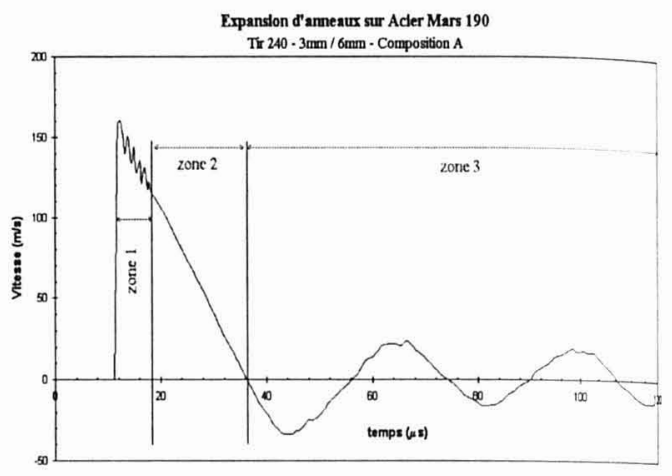

Figure 3 - Courbe de vitesse

\section{1 - Essais classiques}

Les essais dynamiques étant adiabatiques, une correction à été apportée sur la contrainte d'écoulement afin de proposer des courbes isotherme. En effet, en considérant que $90 \%$ de l'énergie de déformation plastique est transformée en chaleur, il est alors possible de quantifier l'élévation de température subie par l'échantillon au cours de l'essai et ainsi de corriger la valeur de la contrainte d'écoulement, connaissant sa sensibilité à la température (valeurs dans les premiers instants de la déformation).

Les coefficients des lois de comportement proposés sont déterminés à l'aide du logiciel RE.N.E. (REsolution of Non-linearly Equation) développé au C.E.A., qui ajuste toutes les données de façon globale, en minimisant l'erreur commise en moyenne quadratique, par l'algorithme de LevenbergMarquardt. Les résultats obtenus sont consignés dans le tableau 1. Les fig. 4 et 5 comparent les résultats des trois modèles aux résultats expérimentaux, sur le cuivre et l'acier.

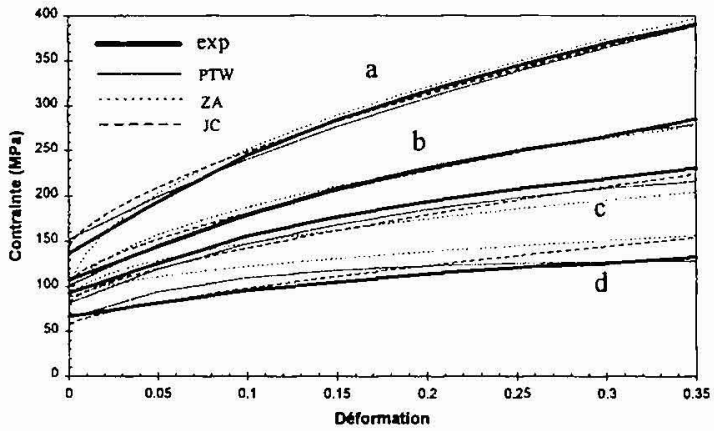

Figure 4 - Comparaison expériences/calculs sur $\mathrm{CuC2}$

a) $\mathrm{T}=20^{\circ} \mathrm{C}, \dot{\varepsilon}=2000 \mathrm{~s}^{-1}$; b) $\mathrm{T}=20^{\circ} \mathrm{C}, \dot{\varepsilon}=0.001 \mathrm{~s}^{-1}$;

c) $\mathrm{T}=400^{\circ} \mathrm{C}, \dot{\varepsilon}=2000 \mathrm{~s}^{-1}$; d) $\mathrm{T}=600^{\circ} \mathrm{C}, \dot{\varepsilon}=2000 \mathrm{~s}^{-1}$.

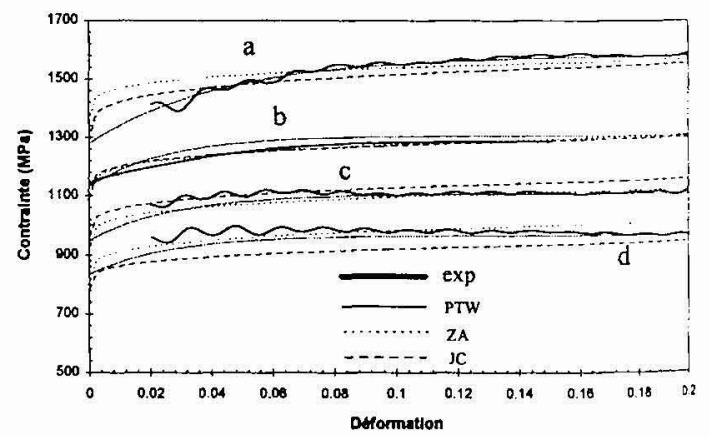

Figure 5 - Comparaison expériences/calculs sur acier Mars 190
a) $\mathrm{T}=20^{\circ} \mathrm{C}, \dot{\varepsilon}=1850 \mathrm{~s}^{-1}$; b) $\mathrm{T}=20^{\circ} \mathrm{C}, \dot{\varepsilon}=0.0001 \mathrm{~s}^{-1}$;
c) $\mathrm{T}=400^{\circ} \mathrm{C}, \dot{\varepsilon}=1750 \mathrm{~s}^{-1}$; d) $\mathrm{T}=600^{\circ} \mathrm{C}, \dot{\varepsilon}=1950 \mathrm{~s}^{-1}$.

\section{2 - Résultats sur expansions d'anneaux}

Les essais sont réalisés sur des anneaux de $3 \mathrm{~mm}$ d'épaisseur, avec des diamètres d'explosif de 6,8 et $10 \mathrm{~mm}$. Les mesures de vitesses d'expansion radiale nous permettent de tracer les courbes $\sigma=f(\varepsilon)$ et $\dot{\varepsilon}=f(\varepsilon)$ pour chaque essai (fig. 6 et 7). La vitesse de déformation décroît au cours de l'essai, jusqu' à 
atteindre une valeur nulle si l'anneau ne rompt pas lors de la phase d'écoulement plastique (cas des essais sur acier Mars 190). Les vitesses de déformations maximales mesurées sont respectivement de 8900 et $8800 \mathrm{~s}^{-1}$ sur l'acier et le cuivre. Ces valeurs peuvent être directement comparés aux résultats expérimentaux obtenus précédemment, afin d'évaluer la sensibilité à la vitesse de déformation de ces matériaux, ainsi qu'à valider les lois de comportement proposées dans cette nouvelle gamme de vitesse (fig. 8 et 9).

Tableau 1 - Coefficients associés à trois lois de comportement $\left(20^{\circ} \mathrm{C}<T<600^{\circ} \mathrm{C}, \dot{\varepsilon}<2500 \mathrm{~s}^{-1}, \varepsilon<0.35\right)$

\begin{tabular}{|c|c|c|c|c|c|c|c|c|c|c|c|c|}
\hline & \multicolumn{5}{|c|}{ Johnson-Cook } & \multicolumn{7}{|c|}{ Zerilli-Armstrong } \\
\hline & $\begin{array}{c}A \\
(\mathrm{MPa})\end{array}$ & $\begin{array}{c}B \\
(\mathrm{MPa})\end{array}$ & $n$ & $C$ & $m$ & $\begin{array}{c}C_{0} \\
(\mathrm{MPa}) \\
\end{array}$ & $\begin{array}{c}C_{1} \\
(\mathrm{MPa})\end{array}$ & $\begin{array}{c}C_{2} \\
(\mathrm{MPa}) \\
\end{array}$ & $\begin{array}{c}C_{3} \\
\left(K^{-1}\right) \\
\end{array}$ & $\begin{array}{c}C_{1} \\
\left(K^{-1} s^{-1}\right) \\
\end{array}$ & $\begin{array}{c}C_{5} \\
(\mathrm{MPa})\end{array}$ & $\bar{n}$ \\
\hline $\mathrm{CuC2}$ & 123,7 & 435,1 & 0,69 & 0,022 & 0,83 & 83,2 & $\cdot$ & 1109 & 0,0035 & 0,000113 & - & - \\
\hline Litt.[1], [3] & 90 & 292 & 0,31 & 0,025 & 1,09 & 62,5 & - & 890 & 0,0028 & 0,000115 & - & - \\
\hline Mars 190 & 1192 & 362,3 & 0,226 & 0,0102 & 1.013 & 602,6 & 1490 & - & 0,0030 & 0,000090 & 299,4 & 0,21 \\
\hline \multirow[t]{3}{*}{ Litt. $[10]$} & 844 & 622,7 & 0,164 & 0,0087 & 1,058 & 138 & 1080 & - & 0,0014 & 0,000036 & 622,7 & 0,164 \\
\hline & \multicolumn{12}{|c|}{ Preston-Tonks-Wallace } \\
\hline & $Y_{0}$ & & & $\mathrm{~S}_{0}$ & $S_{\infty}$ & $\mathrm{k}$ & $\gamma$ & $\theta$ & $p$ & $Y_{1}$ & $Y_{2}$ & $\beta$ \\
\hline $\mathrm{CuC2}$ & 0.00235 & 0.0 & 079 & 0.01738 & 0.00090 & 0.16 & $1.35 \mathrm{e} 9$ & 0.011 & 4 & 0,094 & 575 & $0, \overline{25}$ \\
\hline Litt. [5], [6] & 0.0001 & 0.0 & & 0.0085 & 0.00055 & 0.11 & $1.72 \mathrm{e} 9$ & 0.025 & 2 & 0,094 & 575 & 0,25 \\
\hline Mars 190 & 0,00921 & 0,0 & 689 & 0,01232 & 0,00795 & 0,57 & $1,13 \mathrm{e} 6$ & 0,042 & - & - & - & - \\
\hline
\end{tabular}

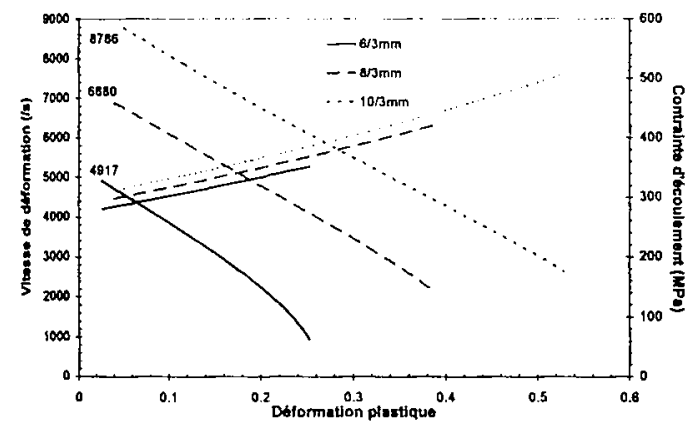

Figure 6-Expansion d'anneaux sur $\mathrm{CuC2}$ : contrainte d'ecoulement et vitesse de déformation en fonction de la déformation

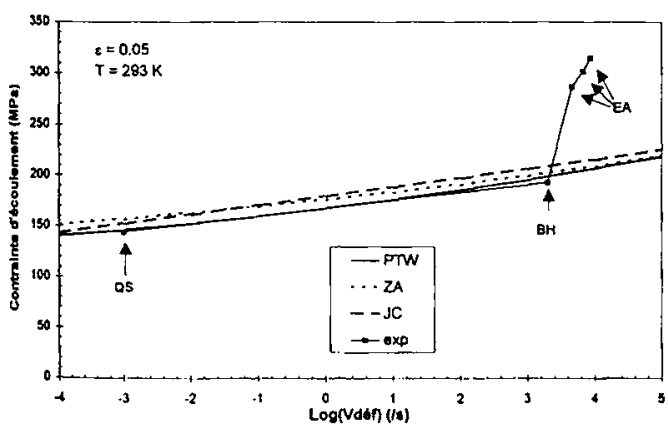

Figure 8 - Contrainte d'écoulement en fonction de la vitesse de déformation ( $\mathrm{CuC2}$ )

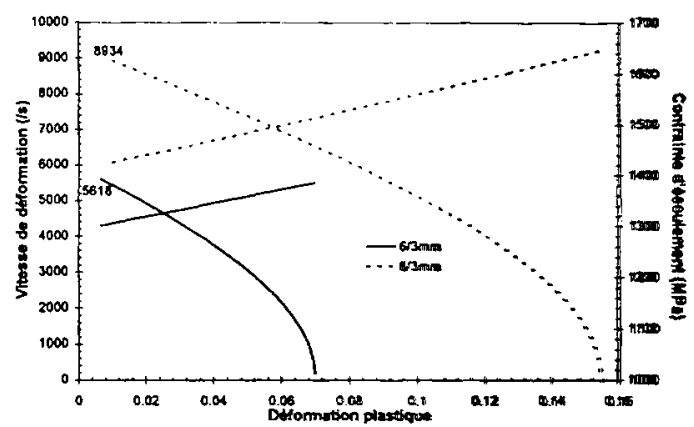

Figure 7 - Expansion d'anneaux sur Mars 190 : comtrainute d'écoulement et vitesse de déformation en fonction de li déformation

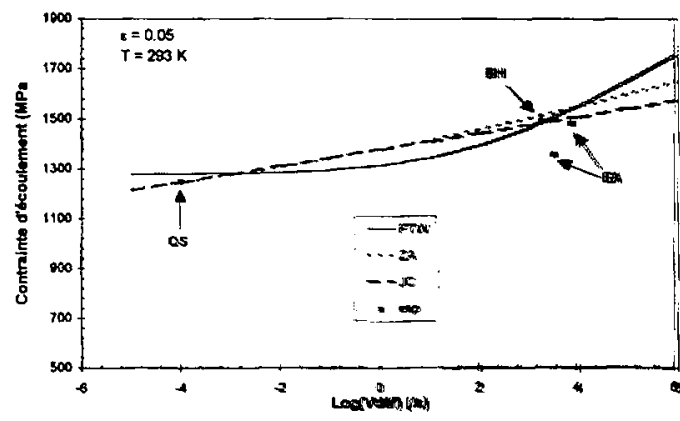

Figure 9 - Contrainte decoulement on fometion to th vitesse de déformation (Mars190)

La fig. 8 nous montre une brusque élévation de la contrainte d'écoulement du cuivre CuC2 pour vitesses de déformation supérieures à $2000 \mathrm{~s}^{-1}$. Seules les données issues des tests d'expansion d'ammeatux permettent de rendre compte de ce phénomène. Dans ce cas, les coefficients des trois loiss de comportement déterminés précédemment doivent être modifiés afin de pouvoir restituer comportement particulier. Le tableau 2 nous donner les valeurs des coefficients madifiés. La fig. 10 
compare les valeurs calculées aux valeurs expérimentales après modification des coefficients. Ces modifications permettent aux modèles de mieux restituer la contrainte d'écoulement aux grandes vitesses de déformation, mais au prix d'une moins bonne précision sur les autres données, à l'exception du modèle PTW. En ce qui concerne l'acier Mars 190, on constate d'après les résultats expérimentaux que ce matériau est très peu sensible à la vitesse de déformation. La fig. 9 montre une bonne corrélation entre la modélisation et l'expérience. Dans ce cas, il n'est pas nécessaire de modifier les coefficients précédemment déterminés.

Tableau 2 - Valeurs modifiées des coefficients de trois lois de comportement pour le cuivre $\mathrm{CuC2}$.

\begin{tabular}{|c|c|c|c|c|c|c|c|c|c|c|c|}
\hline \multicolumn{5}{|c|}{ Johnson-Cook } & \multicolumn{7}{|c|}{ Zerilli-Armstrong } \\
\hline $\begin{array}{c}A \\
(\mathrm{MPa})\end{array}$ & $\begin{array}{c}B \\
(\mathrm{MPa})\end{array}$ & $n$ & $\bar{C}$ & $m$ & $\begin{array}{c}C_{0} \\
(\mathrm{MPa})\end{array}$ & $\begin{array}{c}C_{l} \\
(\mathrm{MPa}) \\
\end{array}$ & $\begin{array}{c}C_{2} \\
(\mathrm{MPa})\end{array}$ & $\begin{array}{c}C_{3} \\
\left(\mathrm{~K}^{-1}\right) \\
\end{array}$ & $\begin{array}{c}C \\
\left(K^{-1} s^{-1}\right)\end{array}$ & $\begin{array}{c}C_{5} \\
(\mathrm{MPa})\end{array}$ & $n$ \\
\hline 129,6 & 291,5 & 0,41 & $0,03 \mathrm{I}$ & 0,71 & 83,2 & - & 1963 & 0,0052 & 0,00023 & - & - \\
\hline \multicolumn{12}{|c|}{ Preston-Tonks-Wallace } \\
\hline$Y_{0}$ & $Y_{\infty}$ & $\mathrm{S}_{0}$ & $S_{\infty}$ & & & $\bar{\gamma}$ & $\theta$ & $\mathrm{p}$ & $Y_{1}$ & $Y_{2}$ & $\beta$ \\
\hline 0.00235 & 0.00079 & 0.01738 & 0.00090 & & & $5 \mathrm{e} 9$ & 0.011 & 4 & 0,303 & 0,389 & 0,25 \\
\hline
\end{tabular}

\section{CONCLUSION}

Cette étude nous a permis d'appliquer le test d'expansion d'anneaux à l'etude de lois de comportement, de deux façons différentes : comme moyen de validation et d'évaluation des modèles, et comme moyen de détermination des coefficients associés à ces derniers. Dans le cas du cuivre $\mathrm{CuC2}$, les résultats obtenus ont permis de mettre en évidence la brusque variation de la sensibilité à la vitesse de déformation pour des vitesses supérieures à $4000 \mathrm{~s}^{-1}$, et de modifier en conséquence les constantes des lois de comportement.

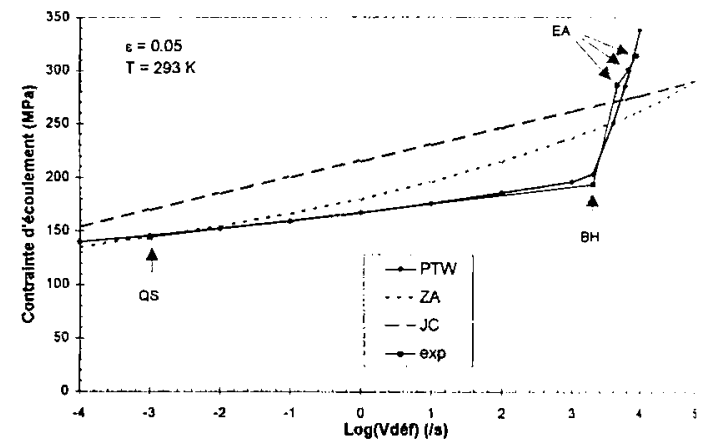

Figure 10 - Contrainte d'écoulement en fonction de la vitesse de déformation ( $\mathrm{CuC2}$ )

A contrario, nous avons montré la faible sensibilité à la vitesse de déformation de l'acier Mars 190 . pour des vitesses inférieures à $8900 \mathrm{~s}^{-1}$; nous avons ainsi vérifier la bonne corrélation calculs/expériences des lois d'écoulement déterminées à partir des données moyennement dynamiques $\left(<2500 \mathrm{~s}^{-1}\right)$.

\section{Remerciements}

Les résultats expérimentaux présentés ici n'auraient pu l'être sans l'aide précieuse de Messieurs P. Antoine, A. Matagne et A. Pérez. Nous remercions également Mr P. Montier de la société GIATi pour la fourniture de l'acier Mars 190.

\section{Références}

[1] Johnson G.R., Cook W.H. $7^{\text {th }}$ Int. Symp. on Ballistics Proceedings, The Hague, 1983, pp. $541-547$.

[2] Johnson G.R., Cook W.H. Engineering Fracture Mechanics, Vol. 21, n 1, 1985, pp.31-48.

[3] Zerilli F.J., Armstrong R.W. J. Applied Phys., 61 (5), March 1987, pp.1816-1825.

[4] Armstrong R.W, Zerilli F.J. J. de Physique, C3, Suppl. au n 9, Tome 49, Sept. 1988, pp. 529-534.

[5] Preston D.L., Tonks D.L., Wallace D.C. Los Alamos National Laboratory, 24/07/1992.

[6] Voce E. J. Inst. Met., Vol. 74, 1947/1948, pp. 537-562.

[7] Carden A.E., Williams P.E., Karpp R.R. Sh. Wave and High Strain Rate Ph. in Metals, Ed. Meyers and LeMurr., Plenum Press, 1981, pp. 23-36.

[8] Goubot N., Naulin G. J. de Phys. IV, Colloque C8, suppl. au J. de Phys. III, Vol. 4, 1994, pp. 189-194.

[9] Goubot N., Th. de Docorat en Sc. et Génie des Matériaux, Ecole des Mines de St Etienne-INPG, 1994.

[10] Gailly B., Thèse de Doctorat, Ecole des Mines de Paris, Décembre 1996. 